\title{
PAIRS OF RINGS WITH A BIJECTIVE CORRESPONDENCE BETWEEN THE PRIME SPECTRA
}

\author{
E. JESPERS and P. WAUTERS
}

(Received 14 February 1991)

Communicated by P. Schultz

\begin{abstract}
Let $A$ be a subring of a commutative ring $B$. If the natural mapping from the prime spectrum of $B$ to the prime spectrum of $A$ is injective (respectively bijective) then the pair $(A, B)$ is said to have the injective (respectively bijective) Spec-map. We give necessary and sufficient conditions for a pair of rings $A$ and $B$ graded by a free abelian group to have the injective (respectively bijective) Spec-map. For this we first deal with the polynomial case. Let $l$ be a field and $k$ a subfield. Then the pair of polynomial rings $(k[X], l[X])$ has the injective Spec-map if and only if $l$ is a purely inseparable extension of $k$.
\end{abstract}

1991 Mathematics subject classification (Amer. Math. Soc.): 13 A 17, 13 B 25, 16 A 03.

Throughout this paper all rings are commutative with identity. For two rings $A \subseteq B$, with the same unity, the $\operatorname{Spec}-\operatorname{map} \varphi$ is the $\operatorname{map} \operatorname{Spec} B \rightarrow \operatorname{Spec} A: P \mapsto$ $P \cap A$. We say that the pair $(A, B)$ has an injective (respectively surjective, bijective) Spec-map if $\varphi$ is injective (respectively surjective, bijective). In general the Spec-map can be arbitrary, but for example if $B$ is integral over $A$ it is well-known that this mapping is surjective. Therefore it is a natural question to study when this mapping is bijective, or injective. A special case of this has been treated by Anderson and Dobbs [1] who considered pairs of rings $(A, B)$

(C) 1993 Australian Mathematical Society 0263-6115/93\$A2.00+0.00

The first named author is supported in part by NSERC-grant 0GP0036631.

The second named author was partially supported by an N.F.W.O.-grant (Belgium) and Memorial University of Newfoundland. He also wishes to thank the Department of Mathematics and Statistics for their warm hospitality. 
for which $\operatorname{Spec} A=\operatorname{Spec} B$, and hence $\varphi$ is the identity mapping. In case $A \neq B$, the latter only happens when $A$ and $B$ have a common unique maximal ideal. However, in general, the condition that the Spec-map is bijective does not imply that $A$ and $B$ are local. For instance, we prove in Proposition 2 that if $k \subseteq l$ are fields, then the polynomial rings $k[X] \subseteq l[X]$ have an injective Spec-map if and only if $l$ is a purely inseparable extension of $k$. Based on this result we can give necessary and sufficient conditions for a pair of rings $A$ and $B$ graded by a free abelian group to have an injective (respectively bijective) Spec-map.

We state the following obvious but useful lemma.

LEMMA 1. Let $A \subseteq B$ be rings.

(1) If for every $b \in B$ there exists an $n>0$ such that $b^{n} \in A$, then the pair $(A, B)$ has a bijective Spec-map.

(2) Let $S$ be a multiplicatively closed subset of elements of $A$ which are regular in $B$. If $(A, B)$ has an injective Spec-map, then so has the pair of localizations $\left(A_{S}, B_{S}\right)$.

(3) If $(A, B)$ has an injective Spec-map and $P$ is an ideal of $B$, then $(A /(P \cap A), B / P)$ has an injective Spec-map.

For a ring $A$ we denote by $A[\mathbf{X}]$ the polynomial ring in the set of variables $\mathbf{X}=\left\{X_{i} \mid i \in I\right\}$. In case $\mathbf{X}$ is a singleton, this ring is simply denoted $A[X]$. Similarly $A\left[\mathbf{X}, \mathbf{X}^{-1}\right]$ denotes the ring of Laurent polynomials. The first and crucial step in our investigations deals with polynomial rings over fields.

PROPOSITION 2. Let $k \subseteq l$ be fields. The following conditions are equivalent:

(1) $(k[X], l[X])$ has an injective Spec-map;

(2) $\left(k\left[X, X^{-1}\right], l\left[X, X^{-1}\right]\right)$ has an injective Spec-map;

(3) $l$ is a purely inseparable extension of $k$. Hence, in each of these cases, the Spec-map is bijective.

PROOF. That (1) is equivalent to (2) is obvious. We now prove that (1) is equivalent to (3). If $l$ over $k$ is purely inseparable then Lemma 1 yields that $(k[X], l[X])$ has a bijective Spec-map. For the converse let $A=k[X]$ and $B=l[X]$. For any $\alpha \in l \backslash k$ the nonzero ideal $B(x-\alpha)$ of $B$ is prime. Hence the intersection is a nonzero prime ideal of $A$ by the injectiveness of the Spec-map. And thus $B(X-\alpha) \cap A=A f$, for some irreducible monic polynomial $f \in A$. Factorizing $f \in B$ in irreducible polynomials, we write $f=(X-\alpha) g_{1} \cdots g_{n-1}$. Clearly, $B g_{i} \cap A=A f$. Since the pair $(A, B)$ has an injective Spec-map each $g_{i}=u_{i}(X-\alpha)$, for some $u_{i} \in l$. Therefore, since $f$ 
is monic, $f=(X-\alpha)^{n}$. Note that $n>1$ since $\alpha \notin k$. Hence we have shown that for every $\alpha \in l \backslash k$ the minimal polynomial over $k$ has multiple roots. The result follows.

Let $G$ be a group and $A=\bigoplus_{g \in G} A_{g}$ a $G$-graded ring. Recall that an ideal $I$ of $A$ is called graded if $I=\bigoplus_{g \in G}\left(I \cap A_{g}\right)$. The largest graded ideal contained in an arbitrary ideal $I$ is denoted $I_{G}$. By $\mathrm{GrSpec} A$ we denote the set of all graded prime ideals of $A$ (for the terminology we refer to [4]). Note that if $G$ is torsion-free abelian, then, for any prime ideal $P$ of $A$, the graded ideal $P_{G}$ is again a prime ideal, and $\mathrm{Gr} \operatorname{Spec} A \subseteq \operatorname{Spec} A$. In this case, if $A$ is a graded subring of the graded ring $B$, that is, $A_{g} \subseteq B_{g}$ for all $g \in G$, we say that the pair $(A, B)$ has an injective GrSpec-map if the restriction of the Spec-map to GrSpec is injective.

Further, for a $G$-graded ring $A$, the ring $Q^{G}(A)$ is the localization of $A$ with respect to the multiplicatively closed set of homogeneous regular elements. In case $A$ is a domain then $Q^{G}(A)$ is a graded field, in the sense that every nonzero homogeneous element is invertible. It is readily seen that every graded field is a twisted group algebra $k^{t}[H]$ for some group $H$ and field $k$. In case $A$ is a domain which is trivially graded, then $Q^{G}(A)$ equals the field of fractions of $A$, which we simply denote by $Q(A)$.

Before stating the main theorem we need two more lemmas.

LEMMA 3. Let $A \subseteq B \subseteq C$ be rings.

(1) If $(A, C)$ has the injective Spec-map, then so has $(B, C)$.

(2) If $C$ is integral over $B$, then $(A, C)$ has an injective Spec-map if and only if both $(A, B)$ and $(B, C)$ have an injective Spec-map.

PROOF. (1) is trivial. Because of the integrality condition (2) follows from the fact that for every prime $p$ of $B$ there exists a prime $P$ of $C$ such that $P \cap B=p$.

LEMMA 4. Let $R=A^{t}[G]$ be a twisted group ring of an arbitrary abelian group $G$ over a ring $A$. If $(A, R)$ has an injective Spec-map, then $G$ is a torsion group.

Proof. Let $T$ be the torsion subgroup of $G, H=G / T$ and $B=A^{t}[T]$. Then $R=B^{t}[H]$ a twisted group ring of $H$ over $B$. Because of Lemma 3.(1) and the assumption, $(B, R)$ has an injective Spec-map. Let $M$ be a maximal ideal of $B$. Since $R=A^{t}[G]=B^{t}[H]$ and $H$ is torsion-free, it follows that 
$M^{t}[H]=R M$ is a prime ideal of $R$. Hence $\bar{B} \subseteq \bar{B}^{t}[H]=B^{t}[H] / M^{t}[H]$ has an injective Spec-map, where $\bar{B}=B / M$. Since $\bar{B}$ is a field and $\bar{B}^{t}[H]$ is a domain, the injective Spec-map property implies that $\bar{B}[H]$ is a field. However, since $H$ is torsion-free, this is only possible if $H$ is trivial, that is, $G=T$. Hence $G$ is torsion.

THEOREM 5. Let $G$ be a free abelian group, and $A$ and $B$ G-graded rings. If $A$ is a graded subring of $B$, then the following conditions are equivalent:

(1) the pair ( $A, B)$ has an injective (respectively, a bijective) Spec-map;

(2) (a) $(A, B)$ has injective (respectively bijective) GrSpec-map, and

(b) for every homogeneous prime ideal $P$ of $B$, the pair $\left(Q^{G}(A / p)\right.$, $\left.Q^{G}(B / P)\right)$ has an injective Spec-map, where $p=P \cap A$;

(3) (a) $(A, B)$ has an injective (respectively bijective) GrSpec-map;

(b) for every homogeneous prime ideal $P$ of $B$, if $B / P$ is non-trivially graded, then $Q(B / P)$ is purely inseparable over $Q(A / p)$, where $p=P \cap A$.

PROOF. Using well-known standard arguments one easily verifies that (1) and (2) are equivalent. Essential is that $P_{G}$ is prime in case $P$ is a prime ideal. Actually for (1) $\Leftrightarrow(2)$ it is sufficient for $G$ to be a torsion-free abelian group.

So to prove the result we only have to show that $(2)(b)$ is equivalent with (3)(b), under the assumption that (2)(a) holds. Note that if (3)(b) holds, then the injective mapping in (2)(b) is automatically bijective. Further, to prove the equivalence, we may assume that $A$ and $B$ are graded fields, that is $A=k^{t}[H] \subseteq$ $B=l^{t}[G]$, where $H$ is a subgroup of $G$. Now if $H$ is a trivial group, that is $A$ is trivially graded, then also $G$ has to be trivial, because otherwise $A$ would be a field and $B$ a non-local domain (since $G$ is free abelian); in contradiction with $(A, B)$ having an injective Spec-map, or $Q(B)$ being purely inseparable over $Q(A)$. On the other hand if both $H$ and $G$ are trivial, then clearly $(A, B)=(k, l)$ has an injective Spec-map. So it is sufficient to show that if $H$ is non-trivial, then $(A, B)$ has an injective Spec-map if and only if $l$ is purely inseparable over $k$ and $G / H$ is $p$-torsion, where $p=\operatorname{char}(k)$ (in case $p=0$, this means $k=l$ and $G=H$ ). The result then follows.

Clearly $l$ over $k$ purely inseparable over $k$ and $G / H$ being $p$-torsion implies that for every $b \in B$ there exists a $n>0$ such that $b^{p^{n}} \in A$. Hence Lemma 1.(1) yields that $(A, B)$ has an injective Spec-map. Conversely assume that $(A, B)$ has an injective Spec-map and $H$ is nontrivial. Since $A=k^{t}[H] \subseteq l^{t}[H] \subseteq l^{t}[G]$, Lemma 3 implies that $\left(l^{t}[H], l^{t}[G]\right)$ has an injective Spec-map. And hence by Lemma $3, G / H$ is a torsion group. Therefore, again by Lemma 3, also 
$\left(k^{t}[H], l^{t}[H]\right)$ has an injective Spec-map.

We now show that $\left(k^{t}[H], l^{t}[H]\right)$ having an injective Spec-map implies that $l$ is purely inseparable over $k$. Since $H$ is free abelian, it is well-known (cf. [4, Exercise 6, page 10]) that $k^{t}[H]=k[H] \subseteq l^{t}[H]=l[H]$, that is, the twisted group algebras are actually group algebras. Let $X$ be a basis for $H$, and pick $y \in X$ (this is possible since $H$ is non-trivial). Let $P$ be the ideal in $l[H]$ generated by all $x-1$, where $x \in X$ and $x \neq y$. Then $k\left[y, y^{-1}\right] \cong k[H] / P \cap k[H]$ and $l\left[y, y^{-1}\right] \cong l[H] / P$. So $\left(k\left[y, y^{-1}\right], l\left[y, y^{-1}\right]\right)$ has an injective Spec-map. Hence Proposition 2 yields that $l$ is purely inseparable over $k$.

Next we show that $\left(l^{t}[H], l^{t}[G]\right)$ having an injective Spec-map implies that $G / H$ is a $p$-torsion group, where $p=\operatorname{char}(k)$. Let $T$ be the subgroup of $G$ containing $H$ such that $T / H$ is a $p$-group and $G / T$ is a $p^{\prime}$-group (that is, not containing elements of order a power of $p$ ). We have to show that $G=T$. Assume this not the case and let $g \in G \backslash T$. Since $l^{t}[H] \subseteq l^{t}[T] \subseteq l^{t}[G]$, Lemma 3 implies that $\left(l^{t}[T], l^{t}[G]\right)$ has an injective Spec-map too. Let $A=$ $l^{t}[T]$ and $B=l^{t}[G]$. Clearly $B=A^{t}[G / T]$, a twisted group ring. Let $M$ be a maximal ideal of $A$. Then $B M=M^{t}[G / T], B / B M=(A / M)^{t}[G / T]$ and $(A / M, B / B M)$ has an injective Spec-map. Since $A / M$ is a field, $B / B M$ is a local ring. On the other hand $A / M$ has characteristic $p$ and $G / T$ is a $p^{\prime}$-group. By Maschke's Theorem (cf. [4, Theorem 4.2]) it follows that $(A / M)^{t}[G / T]$ has trivial Jacobson radical. The fact that $B / B M$ is local therefore means that $B M$ is a maximal ideal, and hence a prime ideal. As before we may assume that $B=l^{t}[G]=l[G]$. Now let $n$ be the smallest number $n$, not divisible by $p$, such that $g^{n} \in T$. Since $\left\{1, g, \ldots, g^{n-1}\right\}$ can be chosen as part of a transversal of $T$ in $G$, it follows that $g-1 \notin B M$ and $g^{n-1}+g^{n-2}+\cdots+g+1 \notin B M$. As $B M$ is a prime ideal, $g^{n}-1=(g-1)\left(g^{n-1}+g^{n-2}+\cdots+g+1\right) \notin B M$. Because $B M \cap A=M$ and $g^{n}-1 \in A$ we obtain that $g^{n}-1 \notin M$. Since this holds for any maximal ideal $M$ of $A, g^{n}-1$ is invertible in $A$, and hence in $B=l[G]$. However this is impossible as $G$ is torsion-free abelian. Hence $G=T$. This finishes the proof.

As an application of the theorem, we give some more examples of pairs with an injective Spec-map.

EXAMPLE 6. Let $\mathbf{X}=\left\{X_{i} \mid i \in I\right\}$ denote an arbitrary set of commuting variables. If $A \subseteq B$ are rings, then the following conditions are equivalent:

(1) ( $A[\mathbf{X}], B[\mathbf{X}])$ has an injective (respectively, a bijective) Spec-map; 
(2) ( $\left.A\left[\mathbf{X}, \mathbf{X}^{-1}\right], B\left[\mathbf{X}, \mathbf{X}^{-1}\right]\right)$ has an injective (respectively, a bijective) Specmap;

(3) $(A, B)$ has an injective (respectively, a bijective) Spec-map and, for every prime ideal $P$ of $B$, the field $Q(B / P)$ is purely inseparable over $Q(A / P \cap$ A).

ProOf. Because of Lemma 1.(2) and Theorem 5 we only have to prove (3) $\Rightarrow$ (1). Assume (3) holds; we check the conditions of Theorem 5.(3). Let $R=A[\mathbf{X}]$ and $T=B[\mathbf{X}]$. One easily verifies that a graded prime ideal $P$ of $T$ is of the form

$$
P=p\left[X_{i} \mid i \in I \backslash J\right]+\sum_{i \in J} X_{i} T,
$$

where $p=P \cap B$ and $J=\left\{i \in I \mid X_{i} \in P\right\}$. Hence it is readily seen that $(R, T)$ has an injective GrSpec-map. Further, $T / P=(B / p)\left[X_{i} \mid i \in I \backslash J\right]$ and $R /(P \cap R)=(A /(p \cap A))\left[X_{i} \mid i \in I \backslash J\right]$. Since $Q(B / p)$ is purely inseparable over $Q(A /(p \cap A))$, so is $Q(T / P)$ over $Q(R / P \cap R)$.

Note that the condition in Example 6.(3) that $Q(B / P)$ is purely inseparable over $Q(A /(P \cap A))$ has to be checked for all prime ideals of $B$ and cannot be weakened to the minimal prime ideals of $B$ (in particular if $B$ is a domain, one is tempted to think that this condition only has to be checked on the zero ideal). For example, let $k$ be a field and $l$ an arbitrary subfield of $k$. Put $A=l+X k[[X]] \subset$ $B=k[[X]]$. Clearly, $A$ and $B$ are local domains with the same maximal ideal $X k[[X]]$. It follows by Proposition 3.8 in $[1]$ that $\operatorname{Spec}(A)=\operatorname{Spec}(B)$. If $P=0$, then $Q(B / P)=Q(B)=Q(A)=Q(A /(P \cap A))$. But if $P=X k[[X]]$, then $Q(B / P)=k$ need not be purely inseparable over $Q(A /(P \cap A))=l$.

The following example is proved in [2].

EXAMPLE 7. Let $\left\{A_{n} \mid n \in \mathbf{N}\right\}$ be an ascending chain of commutative rings and $A=\bigcup_{n \in \mathbf{N}} A_{n}$. Let $R=\bigoplus_{n \in \mathbf{N}} A_{n} X^{n} \subseteq A[X]$ and $T=A_{0}+X A[X] \subseteq A[X]$. Then $(R, T)$ has a bijective Spec-map.

PROOF. This is an easy consequence of Theorem 5. Let $P$ be a graded prime ideal of $R$. If $X \in P$, then $P=p+\left(\bigoplus_{n \geq 1} A_{n} X^{n}\right)$, where $p=P \cap A_{0}$; this follows directly because, if $n \geq 1$, then $\left(A_{n} X^{n}\right)^{2}=\left(A_{n} X^{2 n-1}\right) X \subseteq R X \subseteq P$, hence $A_{n} X^{n} \subseteq P$. If $X \notin P$, then $P=R_{(X)} P \cap R=A\left[X, X^{-1}\right] P \cap R=$ $p\left[X, X^{-1}\right] \cap R$, and $p \in \operatorname{Spec}(A)$. Since the construction of $T$ is a special example of $R$, we have that graded prime ideals of $T$ are of the form $p+X A[X]$ 
with $p \in \operatorname{Spec}\left(A_{0}\right)$, or $p\left[X, X^{-1}\right] \cap T$ with $p \in \operatorname{Spec}(A)$. To check the conditions of Theorem 5 is now straightforward.

In [3] the latter example is extended to polynomial rings in a finite number of variables. The reasoning mentioned above can be extended in a straightforward way to this case.

Theorem 5 does not hold any more for $G$-graded rings where $G$ contains nontrivial torsion elements. This is shown by the following classical example. Let $k(X)$ be the rational function field in one variable over an arbitrary field $k$. Let $n$ be a nonzero positive integer. One easily verifies that $k(X)=k\left(X^{n}\right) 1+$ $k\left(X^{n}\right) X+\cdots+k\left(X^{n}\right) X^{n-1} \cong k\left(X^{n}\right)^{t}\left[C_{n}\right]$, a twisted group ring of the cyclic group $C_{n}$ of order $n$ over the field $k\left(X^{n}\right)$. Hence $k(X)$ is a $C_{n}$-graded ring. Note that $k$ can also be considered as a $C_{n}$-graded ring for the trivial gradation. Clearly, $(k, k(X))$ has an bijective Spec-map, while $k(X)$ is not purely inseparable over $k$.

On the other hand, we have the following.

PROPOSITION 8. Let $A$ be a ring and $G$ an abelian group. Let $A[G]$ be the group ring of $A$ over $G$. Then $(A, A[G])$ has an injective Spec-map if and only if either $\operatorname{char}(A)=0$ and $G$ is trivial, or $\operatorname{char}(A)=p^{n}$ for some prime number $p, n>0$ and $G$ is a p-group.

PROOF. If $\operatorname{char}(A)=p^{n}$ and $G$ is a $p$-group, then for every $\alpha \in A[G]$, there exists $m>0$ such that $\alpha^{m} \in A$. Lemma 1.(1) yields the result. For the converse, suppose that $(A, A[G])$ has an injective Spec-map. By Lemma 4, $G$ is a torsion group. Let $P$ be a prime ideal of $A$, and put $\bar{A}=A / P$. By Lemma 1.(3), $(\bar{A}, \bar{A}[G])$ has an injective Spec-map, and hence, by Lemma 1.(2), $(Q(\bar{A}), Q(\bar{A})[G])$ has an injective Spec-map. Since $Q(\bar{A})$ is a field, this just means that $Q(\bar{A})[G]$ has a unique proper prime ideal. But this only happens (cf. [5]) if either $\operatorname{char}(Q(\bar{A}))=0$ and $G$ is trivial, or $\operatorname{char}(Q(\bar{A}))=p>0$ and $G$ is a $p$-group. Hence, if $G$ is nontrivial, then each quotient $A / P$ has the same prime characteristic $p$. Hence $A$ has characteristic $p^{n}$ for some $n>0$.

COROLlaRY 9. With notation as in Proposition $8,(A, A[G])$ has an injective Spec-map if and only if $(A, A[G])$ has a bijective Spec-map.

COROLLARY 10. Let $k$ be a field and $H$ a proper subgroup of an abelian group $G$. Then $(k[H], k[G])$ has an injective Spec-map if and only if $\operatorname{char}(k)=p>0$ and $G / H$ is a p-group. 
PROOF. Let $W$ be the ideal of $k[G]$ generated by all group algebra elements $h-1, h \in H$, and let $V=W \cap k[H]$, the augmentation ideal of $k[H]$.

If $(k[H], k[G])$ has an injective Spec-map, then, by Lemma 1.(3), so does $(k[H] / V, k[G] / W)$. Since $k[H] / V \cong k$ and $k[G] / W \cong k[G / H]$, Proposition 8 yields that char $(k)=p>0$ and $G / H$ is a $p$-group. Conversely, if the latter conditions are satisfied, then Lemma 1.(1) yields that $(k[H], k[G])$ has indeed an injective Spec-map.

The condition that $(A, B)$ has a bijective Spec-map does not imply that $\operatorname{dim}(A)=\operatorname{dim}(B)$. Here is an example. Let $p$ be a prime number and put $A=\mathbf{Z}_{(p)}$, the localization of $\mathbf{Z}$ at the prime ideal $p \mathbf{Z}, B=\mathbf{Z}_{p}[[t]] \times \mathbf{Q}$ and $C=\mathbf{Z}_{p}((t)) \times \mathbf{Q}$, where $\mathbf{Z}_{p}((t))$ is the ring of Laurent power series. Consider $A$ as a subring of $B$ via the embedding $a \mapsto(\bar{a}, a)$, where $\bar{a}=$ $a(\bmod p)$; and $B$ as a subring of $C$ via the natural embedding. Now, $\operatorname{Spec}(A)=$ $\left\{0, p \mathbf{Z}_{(p)}\right\}, \operatorname{Spec}(B)=\left\{(0, \mathbf{Q}),\left(t \mathbf{Z}_{p}[[t]], \mathbf{Q}\right),\left(\mathbf{Z}_{p}[[t]], 0\right)\right\}$ and $\operatorname{Spec}(C)=$ $\left\{(0, \mathbf{Q}),\left(\mathbf{Z}_{p}((t)), 0\right\}\right.$. Clearly, $(A, C)$ has an injective Spec-map (and hence also $(B, C))$, while $\operatorname{dim}(A)=1$ and $\operatorname{dim}(C)=0$, but $(A, B)$ does not have an injective Spec-map. The latter shows that Lemma 3.(2) does not hold in general.

\section{References}

[1] D. F. Anderson and D. E. Dobbs, 'Pairs of rings with the same prime ideals', Canada. J. Math. 22 (1980), 362-384.

[2] P. J. Cahen and Y. Haouat, 'Spectre d'anneaux de polynômes sur une suite croissante d'anneaux', Arch. Math. 49 (1987), 281-285.

[3] Y. Haouat, 'Spectre d'anneaux de polynômes a plusieurs variables sur une suite d'anneaux', Arch. Math. 50 (1988), 236-240.

[4] D. S. Passman, Infinite crossed products (Academic Press, New York, 1989).

[5] P. Wauters and E. Jespers, 'When is a semigroup ring of a commutative semigroup local or semilocal?', J. Algebra 108 (1987), 188-194.

Memorial University of Newfoundland

St. John's, Newfoundland

Canada A1C 5S7
Economische Hogeschool Limburg and Limburgs Universitair Centrum 3590 Diepenbeek Belgium 